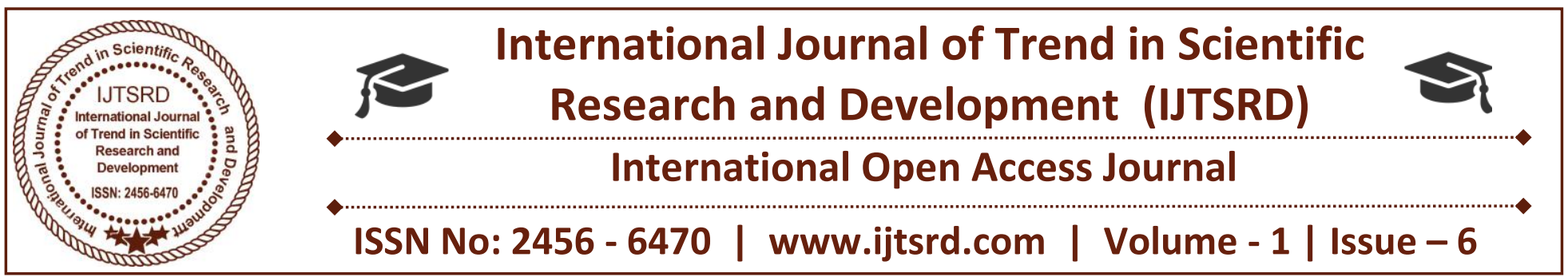

\title{
Transformer Parameter Monitoring and Controlling
}

\author{
Pooja M. Bansod \\ Department of Electronics \& Telecommunication \\ Gondwana University, Gadchiroli
}

\author{
Dinesh V. Rojatkar \\ Department of Electronics \& Telecommunication \\ Gondwana University, Gadchiroli
}

\begin{abstract}
Distribution transformer is one of the most important equipment in power network. Because of, the large amount of transformer distributed over a wide area in power electric systems, the data acquisition and condition monitoring is important issue. The main purpose of this system is distribution transformer monitoring and controlling through IOT. Also, it sends SMS to a central database via the GSM modem for further processing. The idea of on-line monitoring system mixes a global service mobile (GSM) Modem, with chip micro controller and different sensors. It is installed at the site of distribution transformer and the above parameters are recorded using the analog to digital converter (ADC) of the embedded system. The obtained parameters such as voltage, current (over voltage, under voltage, over current) are processed and recorded in the system memory. If any deviation or an emergency situation occurs the system send SMS (short message service) to the mobile phones containing information about the deviation according to some predefined instructions programmed in the micro controller.
\end{abstract}

Keywords: IOT (Internet of Things), GSM, Micro controller, Sensors

\section{INTRODUCTION}

Transformer is a vital part of the transmission and distribution system. Monitoring transformers for problems before they occur can prevent faults that are costly to repair and result in a loss of service. An innovative design to develop a system for monitoring the voltage, current and temperature, oil level of a transformer in a substation and to protect the system from the mentioned parameters based on various application that is used in this paper. A monitoring system can only monitor the operation state or guard against steal the power, and is not able to monitor all useful data of transformers to reduce costs, temperature and over voltage. If the increase in temperature rises higher than the desirable temperature, the monitoring system will protect the distribution by problems. According to above requirements, we need a transformer real time monitoring system to detect all operating parameters operation, and send to the monitoring central in time. The GSM based monitoring of distribution transformer is rather useful as compared to the manual operating system. In case of manual monitoring system it is not possible to monitor the oil level, rise in oil temperature, rise in ambient temperature, load current frequently. After receiving the message of any deviation, it can easily take action immediately to check any failure of distribution transformers. In a distribution network system there are many distribution transformers and connecting each transformer with such system can easily figure out faulty transformer from the message sent to mobile, thereby no need of checking all transformers phase current and voltage and thus it can improve the system in less time. Our system is designed based upon online monitoring of key Operational parameters of transformers can provide useful formation about the health of transformers which will help the utilities to Optimally use their transformer and keep the asset in operation for a longer Period. 


\section{PROPOSED SYSTEM}

Smart grid, the next generation electric power system is actual way of digital transmission of electricity. It is digital technology that allows resourceful two way communications between utility and customers. It provides a system model that will monitor critical parameters such as current, voltage, power and temperature. A smart grid uses digital technology to improve reliability, security and efficiency of the electric system. A system is designed to improve the power quality and reliability of power supply. Using smart grid based sensors like current, voltage and temperature sensor along with IOT wireless communication protocol real time monitoring of Power line parameters like voltage, current, temperature etc. can be carried out. These sensors qualify the remote monitoring of equipment such as transformers and power lines. They are improving the performance and extending the life of grid components to ensure a safe and reliable operation of the electricity network. This system will help us to identify problems before any shattering Failure, thus resulting in a long life service for transformers. In transformer monitoring system we used four sensors for monitoring that is voltage sensor, current sensor, Temperature sensor and oil level sensor. We used power supply to operate micro controller.

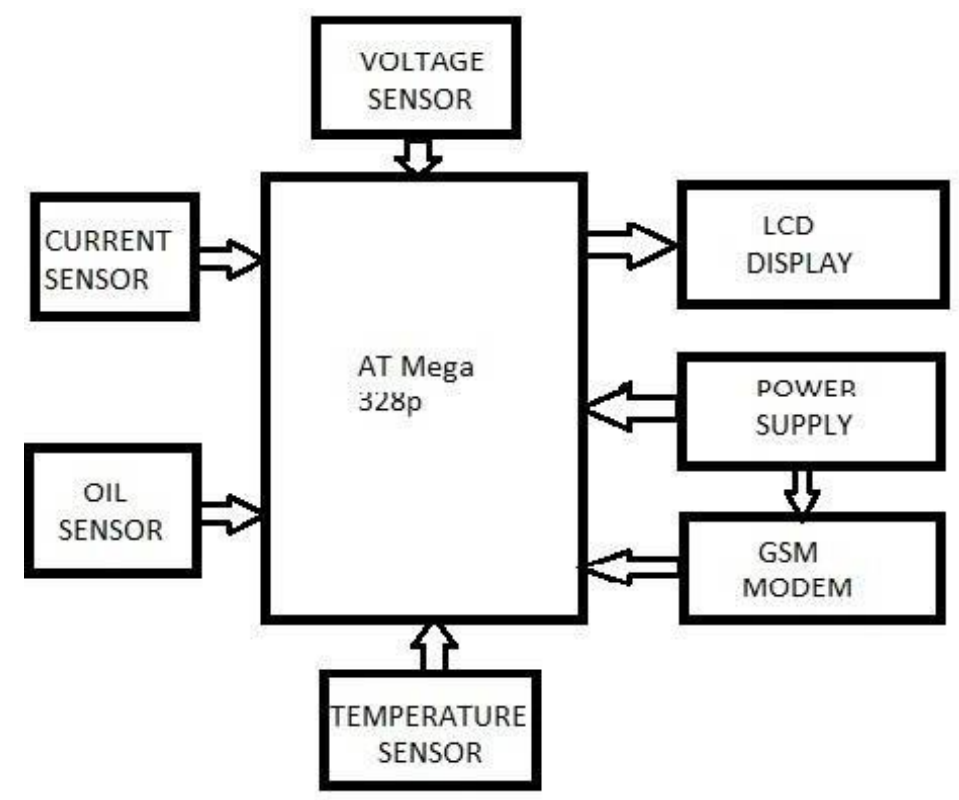

Figure: Block diagram of proposed model

\section{VOLTAGESENSOR}

A voltage sensor is a device which detects the voltage, and generates signal proportional to it. The generated signal could be analog voltage or current or even digital output. It can be then utilized to display the measured voltage in a voltmeter or can be stored for further analysis in a data acquisition system or can be utilized for control purpose. A voltage sensor can in fact determine, monitor and can measure the supply of voltage. It can measure AC or/and DC voltage level. The input to the voltage sensor is the voltage itself and the output can be analog voltage signals, switches, audible signals, analog current level, frequency or even frequency modulated outputs. That is some voltage sensors can provide sine wave or pulse trains as output and others can produce Amplitude Modulation, Pulse Width Modulation or Frequency Modulation outputs.

\section{CURRENT SENSOR}

A current sensor is a device that is used to detect electric current (AC or DC) in a wire, and generates a signal proportional to it. The generated signal could be analog voltage or current or even digital output. It can be utilized to display the measured current in an ammeter or can be stored for further analysis in a data acquisition system or can be utilized for control purpose. A sensor's sensitivity indicates how much the sensor's output changes when the input quantity being measured changes. For instance, the mercury in a thermometer moves 1 $\mathrm{cm}$ then the temperature changes by $1{ }^{\circ} \mathrm{C}$, the sensitivity is $1 \mathrm{~cm} /{ }^{\circ} \mathrm{C}$ (it is basically the slope Dy/Dx assuming a linear characteristic). Some sensors can also affect what they measure; for instance, a room temperature thermometer inserted into a hot cup of liquid cools the liquid while the liquid heats the thermometer. Sensors are usually designed to have a small effect on what the is measured; making the sensor smaller often improves this and may introduce other advantages.[2] Technological progress allows more and more sensors to be manufactured on a microscopic scale as micro sensors using MEMS technology.

\section{OIL SENSOR}

Oil level sensor is a device which is used to check the oil level in the transformer. Due to overheating the oil start to evaporate and the oil level deacrase in the oil level may be dangerous to the transformer. Thus this sensor indicates the level and we get aware about the level. Thus we can look over the oil viscosity also. 


\section{TEMPERATURE SENSOR}

Temperature sensor vary from the simple ON/OFF thermostatic devices which control a domestic hot water heating system to highly sensitive semiconductor types that can be control complex process and control furnace plants. We remember from our school science classes that the movement of molecules and atoms produces heat (kinetic energy) and the greater the movement, the more heat that is generated. Temperature Sensors measure the amount of heat energy of the transformer or even coldness that is generated by an object or system, allowing us to "sense" or detect any physical change to that temperature producing either an analogue or digital output. There are different types of temperature sensor available and all have different characteristics depending upon their actual applications.

\section{CONCLUSION}

The IOT wireless open typical technology is being designated in this project as the energy management and efficiency technology of choice. Employing the system for real time monitoring of power line with an open standard such as iot helps to keep costs down and condensed power consumption. It is clear from the experimentation's that the wireless sensor networks may be successfully employed to smart grids for monitoring resolve. For large scale deployment, cost effective power monitoring system is essential, which requires are liable and low cost WSN mote design.

\section{REFERENCES}

1) Leib fried, $T$, "Online monitors keep Transformers in service", Computer Applications in Power, IEEE, Volume: 11 Issue: 3, July 1998 Page(s):36 42.

2) Chan, W. L, So, A.T.P. and Lai, L., L., "Interment Based Transmission Substation Monitoring", IEEE Transaction on Power Systems, Vol. 14,No. 1, February 1999, pp.293 - 298.

3) Par S. Tenbohlen,T. Stirl, M. Rösner," Benefit of sensors for on-line monitoring system for power transformers"

4) Ansuman Sharma, Rajesh Behura; "GSM based Distribution Transformer Monitoring System", May 2013.

5) Adejumobi, I.G. Adebayo and A.J. Olanipekun.; "Development of Microcontroller-based Electric Power Distribution Automation System", Journal of Innovative Research in Engineering and Sciences, Vol. 2, Issue 1, pp.10-28, March 2011

6) DS1307 $64 \times 8$, Serial, I2C Real-Time Clock, Maxim IntegratedProducts Inc., California, 2008. (2016, Jan 2).

7) Par S. Tenbohlen,T. Stirl, M. Rösner," Benefit of sensors for on-line monitoring system for power transformers"

8) DS1307 $64 \times 8$, Serial, I2C Real-Time Clock, Maxim IntegratedProducts Inc., California, 2008. (2016, Jan 2). 